\title{
Feast : Radical Hospitality in Contemporary Art
}

\section{Marion Lemoult}

\section{(2) OpenEdition \\ Journals}

Édition électronique

URL : http://journals.openedition.org/critiquedart/13358

DOI : 10.4000/critiquedart.13358

ISSN : 2265-9404

Éditeur

Groupement d'intérêt scientifique (GIS) Archives de la critique d'art

Référence électronique

Marion Lemoult, « Feast : Radical Hospitality in Contemporary Art », Critique d'art [En ligne], Toutes les notes de lecture en ligne, mis en ligne le 01 mai 2015, consulté le 22 septembre 2020. URL : http:// journals.openedition.org/critiquedart/13358; DOI : https://doi.org/10.4000/critiquedart.13358

Ce document a été généré automatiquement le 22 septembre 2020.

Archives de la critique d'art 


\title{
Feast : Radical Hospitality in Contemporary Art
}

\author{
Marion Lemoult
}

1 Le catalogue prolonge l'exposition itinérante Feast: Radical Hospitality in Contemporary Art,initiée et présentée par le Smart Museum of Art (Chicago). Présentée en 2012, elle a depuis voyagé au Blaffer Art Museum (Houston), au SITE (Santa Fe) et poursuivra sa route jusqu'en 2015 à la Gund Gallery (Gambier) et au Weisman Art Museum (Minneapolis). Dans une mise en perspective historique, l'ouvrage, introduit par Stephanie Smith (p. 12-21), restitue les œuvres présentées et les actions expérimentales qui se sont jouées au cœur des espaces, mais aussi avec le public tout au long de l'exposition.

2 Le festin, ou le repas, est mis en exergue pour la première fois. Rituel sociétal, il permet de rassembler, d'exclure, d'investiguer les relations qui s'attirent, se repoussent et s'échangent entre hôte et invité. Les pratiques artistiques ayant investi ce paradigme tel un matériau esthétique sont multiples et variées. Feast restitue les œuvres de plus de trente artistes, et propose de faire remonter les origines de ces pratiques aux années 1930, avec les futuristes italiens, notamment F.T. Marinetti et son Manifeste de la Cuisine Futuriste (p.32-39). Les artistes présentés sont rassemblés selon des critères chronologiques qui permettent de différencier les propositions: celle des décennies 1960 et 1970 (p. 50-153), où différentes figures de l'art ont travaillé sur le festin en tant qu'entité « conceptuelle et performative » (Marina Abramović et Ulay, Alison Knowles, Suzanne Lacy, Tom Marioni, Gordon Matta-Clark, Allen Ruppersberg, Bonnie Ora Sherk, Barbara T. Smith, Daniel Spoerri) ; puis celle qui voit de plus jeunes artistes ou collectifs d'artistes internationaux, depuis les années 1990 jusqu'à nos jours (p. 156-345), travailler leurs festins vers une portée "sociale et critique " (Sonja Alhäuser, Mary Ellen Carroll, Fallen Fruit, Theaster Gates, InCUBATE, Mella Jaarsma, Lee Mingwei, Laura Letinsky, Mildred's Lane, Julio Cesar Morales, motiroti, The National Bitter Melon Council, Ana Prvacki, Sudsiri Pui-Ock, Michael Rakowitz, Ayman Ramadan, Red76, David Robbins, Rirkrit Tiravanija). 
3 Plus qu'un catalogue, il s'impose comme un ouvrage majeur pour l'histoire de l'art. Il réunit à la fois les essais relatant la ligne curatoriale de l'exposition, des entretiens et des textes inédits d'artistes, d'auteurs proposés dans une lecture historique, mais aussi les traces photographiques d'installations éphémères et des processus de création. Un large espace est dédié à chacun des artistes représentés. Permettant de saisir les pratiques dans leur singularité, cette publication traduit la radicalité et la multiplicité à laquelle peut recouvrir cette forme d'hospitalité. Dans une société toujours plus rapide et segmentée, la simple action de partager un repas se transforme en un engagement critique face à la culture dans laquelle celui-ci est mis en œuvre. 